\title{
Simulação do transporte de partículas em suspensão e de poluentes dissolvidos pelo escoamento em superfícies impermeáveis: uma contribuição ao saneamento urbano
}

\author{
Simulation of the transport of suspended particles and dissolved pollutants \\ by runoff on impervious surfaces: a contribution to urban sanitation
}

\section{Jéssica Giacchetto Felice', Alexandre Silveira ${ }^{2 *}$, Jorge Manuel Pereira Guieiro Isidoro ${ }^{3}$, Flávio Aparecido Gonçalves ${ }^{2}$, Antônio Marciano da Silva ${ }^{4}$}

$\square$

\begin{abstract}
RESUMO
Um dos impactos da urbanização no meio ambiente é a alteração do processo de transformação da chuva em escoamento superficial, levando à alteração da qualidade dos recursos hídricos. Com este trabalho, objetivou-se simular o transporte de partículas em suspensão e de poluentes dissolvidos, transportados pelo escoamento, sobre uma superfície impermeável em escala de laboratório. O modelo experimental consistiu de um simulador para a aplicação de chuva artificial sobre uma superfície acrílica impermeável com $8,40 \mathrm{~m}^{2}$, na qual foram definidas quatro posições para a distribuição do cloreto de sódio e da areia fina, simulando fontes difusas de poluição. Os resultados indicaram diferenças entre os tempos de pico do transporte de poluentes dissolvidos e em suspensão, em função da posição desses. O efeito de first flush foi verificado para os poluentes dissolvidos independentemente da posição da fonte, enquanto para os poluentes transportados em suspensão o mesmo efeito só foi verificado quando a fonte estava mais próxima da saída da superfície impermeável.

Palavras-chave: chuva artificial; processo chuva-escoamento; transporte de partículas; transporte de poluentes; modelagem física.
\end{abstract}

\begin{abstract}
One of the impacts of urbanization on the environment is the changes in the rainfall-runoff process, causing changes in water resources quality. The objective of this project was to simulate the transport of suspended particles and pollutants dissolved on runoff, an impermeable surface at laboratory scale. The experimental model consisted on a simulator for the application of artificial rainfall on an impermeable surface with $8,40 \mathrm{~m}^{2}$, in which sodium chloride and fine sand were distributed in four defined regions, simulating sources of diffuse pollution. The results showed differences between peak transport times of dissolved and suspended pollutants, depending on their position. The first-flush effect was observed for the dissolved pollutants independently of the source position, whereas for the pollutants transported in suspension the same effect was only found when the source was closer to the outlet of the impervious surface.
\end{abstract}

Keywords: artificial rainfall; rainfall-runoff process; particle transport; pollutant transport; physical modelling.

\section{INTRODUÇÃO}

As cidades são a maior interferência do homem no ambiente natural (MORAES et al., 2014). A alta taxa de urbanização e, consequentemente, a impermeabilização do solo têm contribuído para o aumento de inundações e a diminuição da qualidade de corpos hídricos receptores do escoamento superficial urbano (NASCIMENTO; HELLER, 2005). Em locais com alto índice de ocupação, as áreas impermeáveis, exemplificadas pelo sistema viário e estacionamentos, podem representar até $30 \%$ da área de drenagem de uma bacia hidrográfica urbana (CASTRO et al., 2013). Decorrente da drenagem

\footnotetext{
'Universidade Federal de Alfenas (UNIFAL) - Alfenas (MG), Brasil. 2Professor Associado da UNIFAL - Alfenas (MG), Brasil.

${ }^{3}$ Professor Adjunto da Universidade do Algarve (UAlg) - Faro, Portugal.

${ }^{4}$ Professor Visitante Nacional Sênior da UNIFAL - Alfenas (MG), Brasil.

*Autor correspondente: alexandre.silveira@unifal-mg.edu.br

Recebido em: 28/12/16 - Aceito em: 08/05/17 - Reg. ABES: 173838
} 
das áreas impermeáveis, os poluentes depositados na superfície da bacia são carreados pelo escoamento superficial, levando à ocorrência do que é usualmente descrito como "poluição difusa". Em geral, as pesquisas concentram-se na variação temporal dos parâmetros de qualidade da água, induzindo incertezas e aumentando o risco de falha nas estruturas de controle. Dessa forma, a variação espacial da poluição difusa fundamenta o gerenciamento adequado dos deflúvios, possibilitando melhores condições de uso e aumentando o número de estudos na área (GOMES, 2014).

O impacto ambiental das cidades não é limitado ao seu ambiente local, tendo alcance também na esfera regional (UNITED NATIONS DEPARTMENT OF ECONOMIC AND SOCIAL AFFAIRS, 2011). Em termos locais, em áreas de drenagem urbana parte da água que infiltrava no solo passa a compor o escoamento superficial, em consequência de alterações nos processos do ciclo hidrológico, como na interceptação e na infiltração (MILLER et al., 2014; LIU et al., 2015). Essas perturbações são visíveis nos hidrogramas do escoamento superficial, os quais tendem a apresentar um menor tempo e maior vazão de pico, quando comparados às condições de pré-urbanização.

Além das mudanças no comportamento do processo chuva- escoamento, na esfera regional, observa-se a variação da qualidade da água em corpos receptores (EGODAWATTA; THOMAS; GOONETILLEKE, 2009; O’DRISCOLL et al., 2010; VINAGRE; LIMA; LIMA JUNIOR, 2015). Essas alterações podem ser influenciadas pelo clima, características da bacia e infraestrutura de drenagem (TANG et al., 2013). Porém, estudos recentes têm se concentrado no aumento da geração e do transporte de sedimentos e poluentes por superfícies impermeáveis e atividades urbanas, os quais trazem mudanças químicas, físicas e biológicas ao meio natural (GRIMM et al., 2008; LEDFORD; LAUTZ, 2015).

Em áreas rurais, menos urbanizadas, o sedimento transportado é resultante do processo de desagregação, transporte e deposição das partículas formadoras do solo (LIMA; SOUZA; SINGH, 2008) pela ação do escoamento superficial sobre o solo exposto, intensificando os processos erosivos (POLETO, 2008). A caracterização desse sedimento é importante para predizer os possíveis impactos ambientais, bem como compreender o processo de acumulação de poluentes em função da geração de sedimentos na bacia, já que uma grande quantidade de poluentes encontrados no escoamento superficial é transportada em associação aos sedimentos (VAZE; CHIEW, 2003; DENG; LIMA; SINGH, 2005; BI; MONETTE; GASPERI, 2015).

Em áreas urbanizadas, a deposição atmosférica, o desgaste da pavimentação, os resíduos de construção civil e a descarga de esgoto sanitário são exemplos de fontes geradoras de carga de poluentes (ALI; BONHOMME; CHEBBO, 2016). Como consequência desse impacto ambiental, tem-se o aumento da concentração de sólidos totais dissolvidos dos corpos hídricos receptores, sendo que esses fatores podem estar relacionados ao tipo de uso do solo e a atividade antrópica desenvolvida (OMETO et al., 2000; PAUL; MEYER, 2001). Assim, devido à preocupação com a poluição hídrica decorrente do processo de urbanização, o cloreto de sódio tem sido utilizado como indicador da existência de outros poluentes transportados em solução pelo escoamento superficial (HERNÁNDEZ, 2009; PINHEIRO et al., 2013; KEMERICH et al., 2013).

As fontes difusas são caracterizadas por apresentarem múltiplas fontes de descarga e, durante os eventos de precipitação, os poluentes acumulados são carreados pelo escoamento superficial. A intensidade e a duração dos eventos de chuva influenciam na quantificação da carga de poluentes, sendo que, no início do escoamento, as cargas são rapidamente lavadas, apresentando altas concentrações e, no decorrer do evento de precipitação, essas concentrações tendem a diminuir até o esgotamento (LIMA, 2001; KIM et al., 2005). A esse fenômeno dá-se o nome de efeito de first flush. A forma como os poluentes são transportados pelo escoamento - particulados ou dissolvidos - determina a facilidade de serem movidos na superfície de captação e transportados pela rede de coleta; e.g., partículas de menor diâmetro são mais fáceis de serem transportadas (FLETCHER; ANDRIEW; HAMEL, 2013).

Nessa perspectiva, a modelagem física é uma ferramenta importante para a análise e compreensão da resposta hidrológica das bacias (AKSOY et al., 2012; CARVALHO, 2014), bem como da dinâmica de sedimentos e poluentes associada ao escoamento superficial. Associados aos modelos físicos, os simuladores de chuva em escala laboratorial têm sido largamente utilizados no estudo do processo chuva-escoamento e no monitoramento das variáveis envolvidas neste processo (MUTCHLER; MOLDENHAUER 1963; ISERLOH et al., 2013; SILVEIRA et al., 2017). A modelagem física com chuva artificial permite superar as limitações impostas pelo ambiente natural, como a impossibilidade de se controlar a distribuição espacial e temporal da chuva, o elevado custo para monitoramento do escoamento superficial e a topografia da área (SPOHR et al., 2015). Ainda, essas ferramentas têm sua aplicação justificada pelo controle das condições ambientais, das características da precipitação e pela reprodutibilidade dos experimentos em pequenos intervalos de tempo (CLARKE; WALSH, 2007; ABUDI; CARMI; BERLINER, 2012).

Objetivou-se com este trabalho simular experimentalmente o transporte de poluentes difusos, em uma superfície impermeável, tendo como fatores de variação a localização da fonte de poluição em relação ao exutório e a forma de transporte: em suspensão (partículas de areia) e dissolvidos (cloreto de sódio). 


\section{MATERIAIS E MÉTODOS}

\section{Configuração experimental}

A montagem experimental utilizada foi adaptada de Reis (2015) e consiste em um simulador de chuva e em uma superfície acrílica impermeável. O escoamento gerado pela aplicação de chuva artificial escoa pelas vertentes da superfície e por uma calha metálica posicionada no centro do modelo experimental. A coleta e a quantificação do escoamento e do material transportado em suspensão e dissolvido foram realizadas na seção final da calha, i.e., no exutório da superfície impermeável (Figura 1).

\section{Simulador de chuva}

O simulador de chuva utilizado é composto por três bocais do tipo cone completo (FullJet 1/4HH - 14W, Spraying System Co.). Uma válvula solenoide instalada próxima dos bocais foi utilizada para permitir a abertura e o fechamento automático do sistema hidráulico. Um manômetro digital instalado no recalque da bomba permitiu o monitoramento da pressão, garantindo que os experimentos fossem realizados sob as mesmas condições de pressão/vazão. Os bocais foram instalados em uma tubulação de cloreto de polivinil (PVC) com 12,5 mm de diâmetro, disposta no sentido longitudinal do modelo experimental, sendo um bocal na posição central e dois outros nas extremidades, distantes de 1,3 m entre si. O bocal central estava posicionado a $2,5 \mathrm{~m}$ na vertical em relação à superfície impermeável. A alimentação e a pressuriza ção da tubulação e dos bocais foram feitas com um conjunto motor-bomba (potência de 745,7 W) conectado a um reservatório por uma tubulação de sucção em material PVC e com diâmetro de $25 \mathrm{~mm}$. A capacidade do reservatório é de 310 litros e o nível d'água no seu interior foi mantido sempre constante. Durante os ensaios, a pressão na tubulação medida pelo manômetro foi igual a $0,16 \mathrm{MPa}$ com os três bocais em funcionamento.

\section{Superfície impermeável}

A superfície impermeável corresponde a duas placas planas, cada uma com dimensão de 4,0 × 1,0 m, em polimetil-metacrilato PPMA (acrílico) com $6 \mathrm{~mm}$ de espessura, dispostas em formato de "V", ou seja, declive longitudinal e transversal ajustável (Figura 1). A escolha do material justifica-se pela simplicidade de uso e absorção nula, de forma a minimizar as perdas de água precipitada.

Uma calha metálica $(4,00 \mathrm{~m}$ de comprimento $\times 0,10 \mathrm{~m}$ de largura $\times 0,09 \mathrm{~m}$ de profundidade) foi posicionada na região central $\mathrm{da}$ superfície acrílica para a captação e o direcionamento do escoamento até a saída do modelo físico (exutório da superfície impermeável). Considerando as dimensões das duas placas e da calha, a área total da superfície de escoamento é de $8,40 \mathrm{~m}^{2}$.

\section{Distribuição espacial da precipitação}

Para caracterizar a distribuição espacial da precipitação foram realizados três ensaios aplicando-se chuva durante 15 minutos (em cada ensaio) sobre a superfície impermeável, com declividade nula nos dois sentidos. Para quantificar a precipitação em cada teste, 200 coletores foram distribuídos de forma equidistante por toda área de captação. Os valores das lâminas precipitadas em cada posição identificada na Figura 1 (lado direito) foram convertidos em intensidade média de precipitação, cuja uniformidade de distribuição foi expressa pelo Coeficiente de Uniformidade de Christiansen (CUC) (CHRISTIANSEN, 1941). A distribuição espacial resultante, obtida por krigagem, está apresentada na Figura 2, sendo que sobre toda a área da superfície, a intensidade de precipitação média foi de $69,02 \mathrm{~mm} \cdot \mathrm{h}^{-1}$ e o CUC de $77,68 \%$.
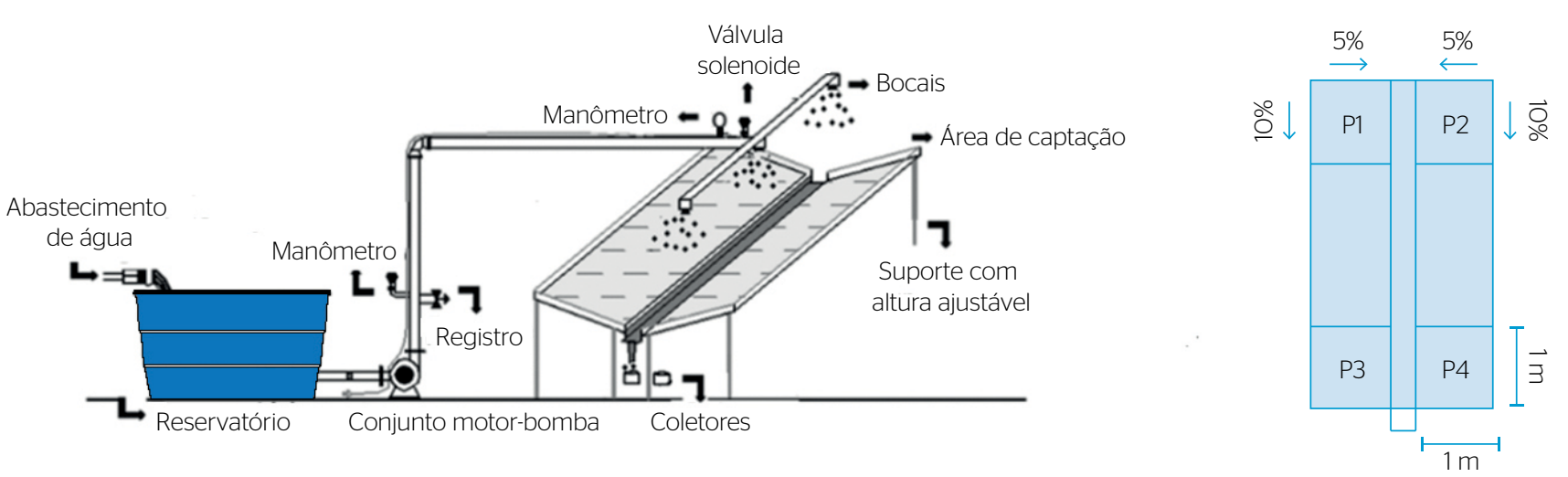

Figura 1 - Aparato experimental - simulador de chuvas, superfície impermeável e coletores do escoamento (esquerda); Identificação das posições em que foram colocados a areia fina e o cloreto de sódio na superfície acrílica impermeável (direita). 


\section{Delineamento experimental}

O estudo abrangeu a avaliação do transporte de poluentes difusos, em um arranjo fatorial: posição da fonte de poluente em relação ao exutório da área de drenagem (quatro posições); forma de transporte: em suspensão - partículas de areia e dissolvido - cloreto de sódio (dois materiais), resultando assim em oito combinações.

Para cada combinação dos fatores de variação foram realizados cinco ensaios a fim de permitir a análise estatística dos resultados experimentais. Esse delineamento resultou em 20 ensaios para cada tipo de poluente analisado (cinco em cada posição, P1 a P4), perfazendo um total de 40 ensaios. Nos ensaios realizados, as declividades da superfície impermeável foram $10 \%$ na longitudinal e 5\% na transversal.
Para assegurar a semelhança da condição inicial de cada ensaio, previamente procedeu-se à aplicação de uma chuva artificial durante dois minutos, seguindo-se um intervalo de cinco minutos até o início de cada ensaio. Esse intervalo de tempo foi utilizado para a distribuição do material (areia ou cloreto de sódio) de forma uniforme sobre a superfície impermeável de cada uma das quatro posições.

Cada ensaio teve a aplicação de chuva artificial com intensidade média de $69,02 \mathrm{~mm} \cdot \mathrm{h}^{-1}$ e 3 minutos de duração, sendo que o deflúvio foi quantificado no exutório da calha metálica. Vale referir ainda que, nos ensaios, as medições se iniciaram no momento em que um filete contínuo de água começou a escoar no exutório da calha e que o final

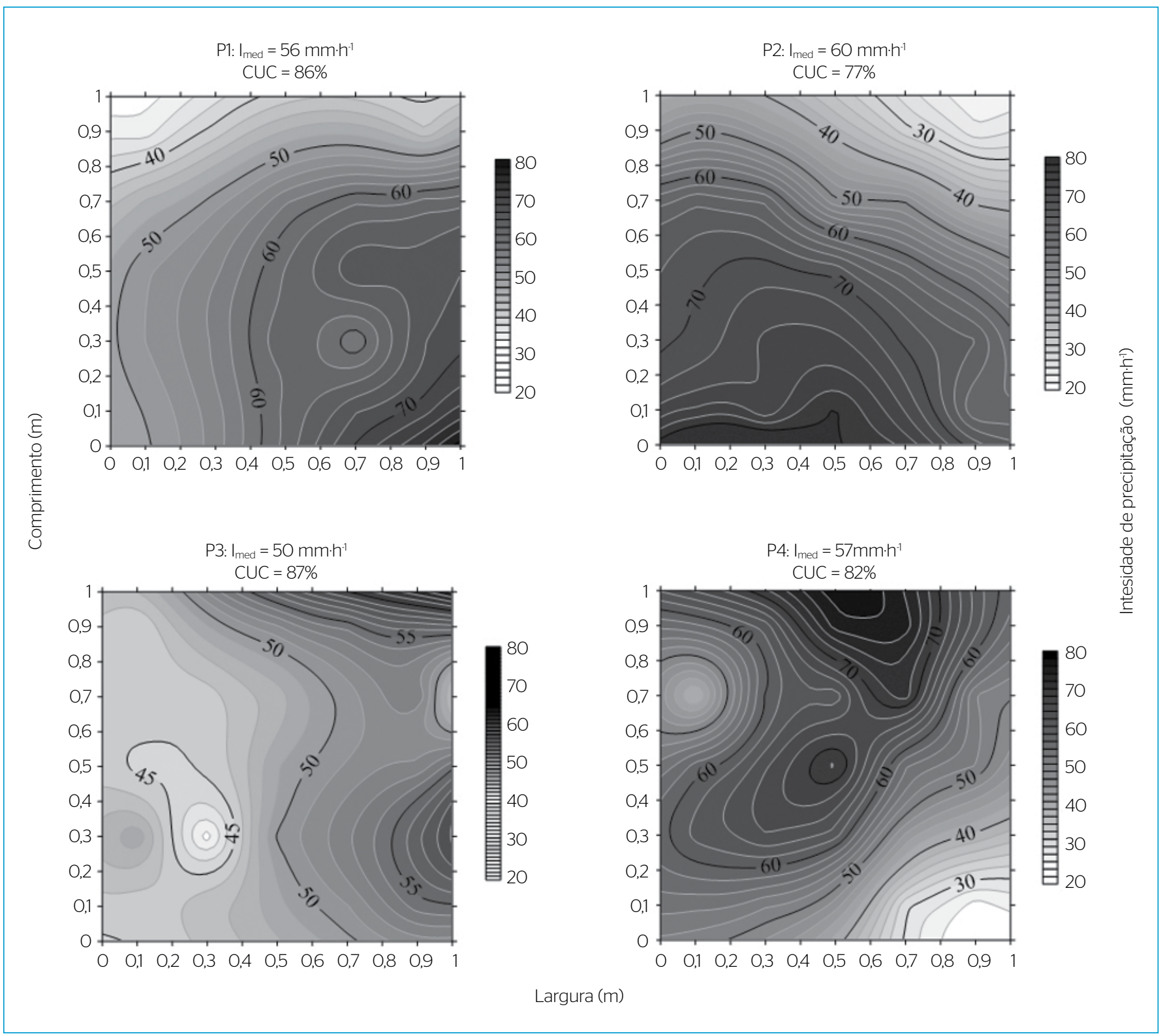

Figura 2 - Intensidade de precipitação média $\left(I_{\text {med }}\right)$, coeficiente de uniformidade de Christiansen (CUC) e distribuição espacial da precipitação para cada posição do modelo experimental. 
do escoamento superficial foi considerado no instante em que esse filete deixou de ser contínuo e passou a intermitente (gotejar).

\section{Ensaios com partículas em suspensão}

Foram distribuídos uniformemente sobre a superfície de cada posição em avaliação (P1 a P4), 20,0 g de areia fina (areia quartzosa industrial AQ 90-500 SE, Mineração Mjundu'), previamente peneirada, com granulometria entre 0,106 e $0,425 \mathrm{~mm}$. O deflúvio foi quantificado no exutório do modelo físico em dez instantes distintos, recorrendo a recipientes plásticos, sendo os pontos médios dos tempos de coleta iguais a $13 ; 15,5 ; 21 ; 26 ; 31 ; 105,5 ; 145,5 ; 185,5 ; 223 ; 263 ; 313$ s, abrangendo a ascensão, o tempo de pico e a recessão do hidrograma do escoamento superficial. As dez amostras coletadas durante o ensaio passaram pelo processo de filtração a vácuo, utilizando filtros com abertura de poros entre 4 e $12 \mu \mathrm{m}$ e, posteriormente, foram submetidas a secagem sob temperatura de $100^{\circ} \mathrm{C}$ durante 24 horas para a determinação da massa de partículas transportadas. Com as informações obtidas do deflúvio construíram-se os hidrogramas e, com as massas de material em suspensão transportadas, os particulogramas.

\section{Ensaios com cloreto de sódio}

Da mesma forma que nos ensaios com material em suspensão, foram distribuídos uniformemente na superfície de cada posição em avaliação (P1 a P4) 10,0 g de cloreto de sódio. Um sensor (Vernier Conductivity Probe $)$, posicionado no final da calha (exutório), foi utilizado para realizar medições de condutividade elétrica a cada segundo durante o deflúvio.

Para a conversão dos valores de condutividade elétrica em concentração de sólidos totais dissolvidos, foi realizado um teste de calibração cujo resultado é apresentado na Figura 3. Para esse teste foi utilizado um litro de água amostrado do reservatório de alimentação do simulador de chuva e mediu-se a condutividade elétrica para cada miligrama de cloreto de sódio adicionado à solução. Para o cálculo da

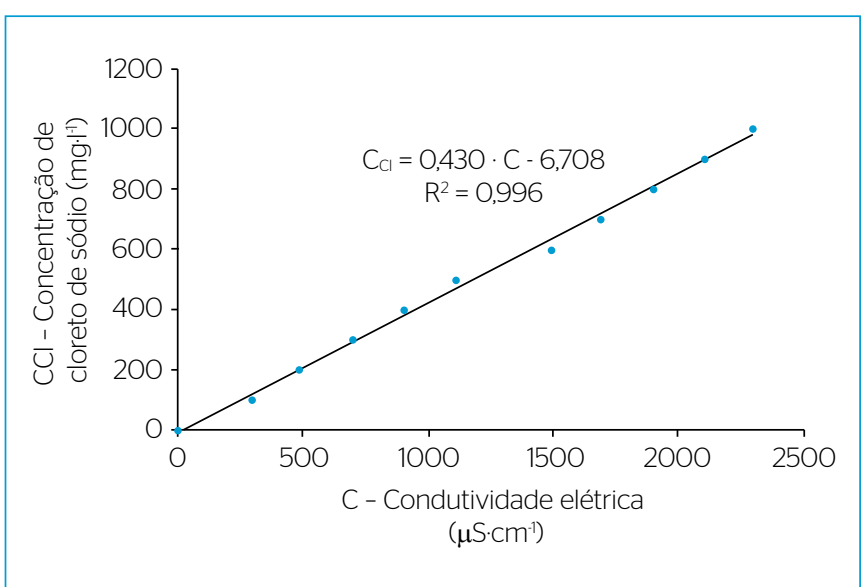

Figura 3 - Curva de calibração da relação entre condutividade elétrica $\left(\mu \mathrm{S} \cdot \mathrm{cm}^{-1}\right)$ e concentração de sólidos totais dissolvidos $\left(\mathrm{mg} \cdot \mathrm{l}^{-1}\right)$. relação entre a condutividade elétrica e a concentração de sólidos totais dissolvidos nos experimentos foi utilizada a equação apresentada na Figura 3, descontando o valor da condutividade elétrica já presente na água do reservatório, para que, então, fosse medida apenas a condutividade induzida pela concentração de cloreto de sódio. Com a análise do transporte de massa durante o escoamento superficial foi possível a construção dos polutogramas.

\section{RESULTADOS E DISCUSSÃO}

Na Figura 4 estão apresentados os hidrogramas médios dos cinco ensaios realizados com a fonte difusa de material particulado (areia), distribuído em cada uma das quatro posições estudadas, bem como o desvio-padrão para cada momento de quantificação do deflúvio.

Pode-se observar na Figura 4 que, considerando o desvio-padrão, a posição dos poluentes não interferiu na forma dos hidrogramas nem nas vazões de pico (patamar). O volume escoado, obtido pela integração de cada hidrograma, também não apresentou diferenças considerando as

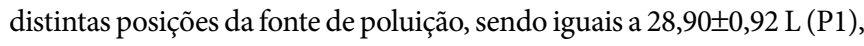

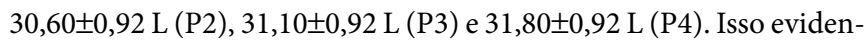
cia que a presença de areia na superfície impermeável não interfere no escoamento superficial.

Na Figura 5 estão apresentados os particulogramas médios (descarga mássica de partículas em função do tempo) para todas as posições (P1 a P4) da fonte de poluição (areia). Pode-se observar que o instante em que o transporte de areia atinge seu valor máximo é superior a quatro vezes para as posições que estão mais distantes do exutório quando comparado àquelas mais próximas do mesmo.

O valor de pico da descarga mássica obtido para P3 e P4 (0,20 e 0,26 g. $\mathrm{s}^{-1}$, respectivamente) foi, aproximadamente, o dobro em relação à P1 e P2 (0,13 e 0,13 g.s ${ }^{-1}$, respectivamente). Observa-se ainda que a posição da fonte de poluição (partículas de areia) influencia não só

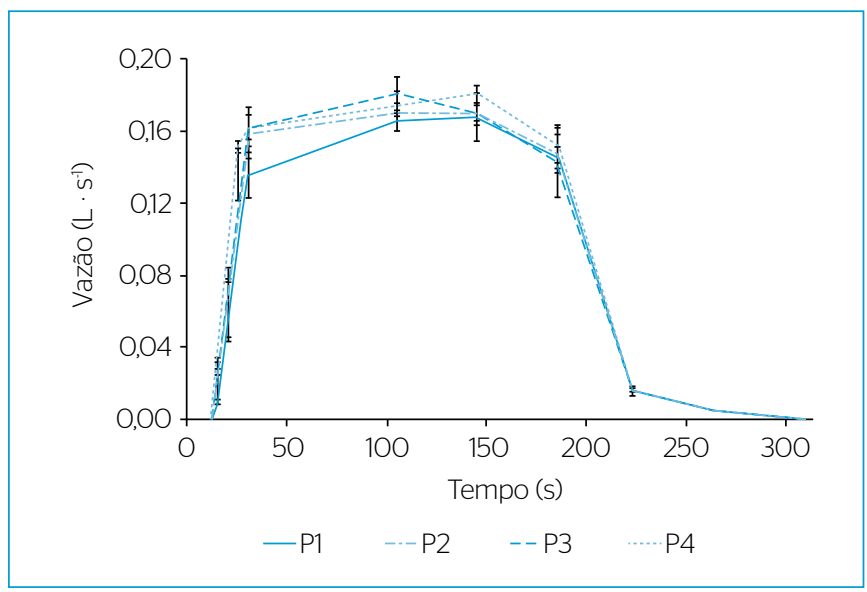

Figura 4 - Hidrogramas médios observados para as quatro posições estudadas (P1, P2, P3 e P4). 
no tempo em que ocorre o valor do pico de descarga, mas também o valor do pico (descarga mássica máxima).

Na Figura 6 estão apresentados os polutogramas médios (descarga mássica de cloreto de sódio em função do tempo) para as quatro posições em avaliação da superfície impermeável (P1 a P4). Observa-se a diferença na forma dos polutogramas para as diferentes posições da fonte de poluição (cloreto de sódio) na superfície. Nas posições mais próximas do exutório, os polutogramas apresentam picos pronunciados,

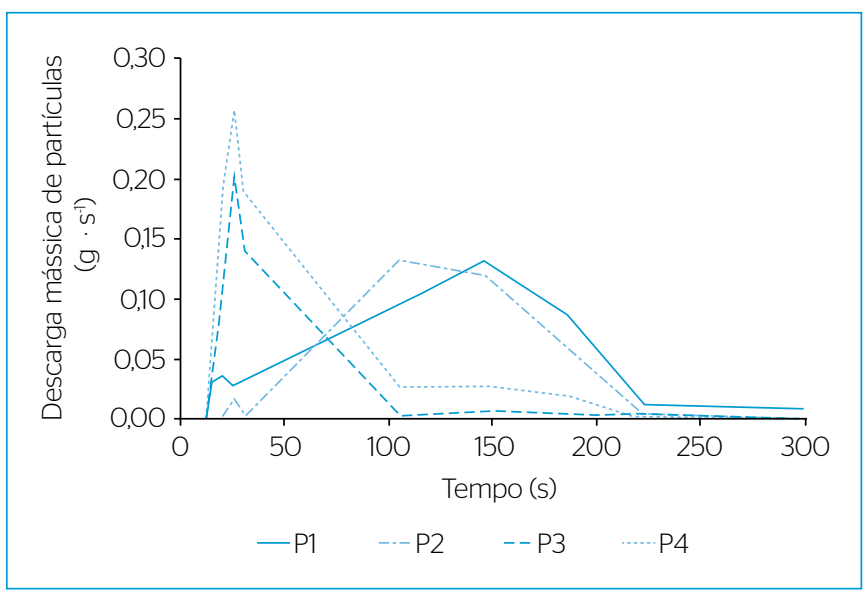

Figura 5 - Particulogramas médios observados para as quatro posições estudadas (P1, P2, P3 e P4).

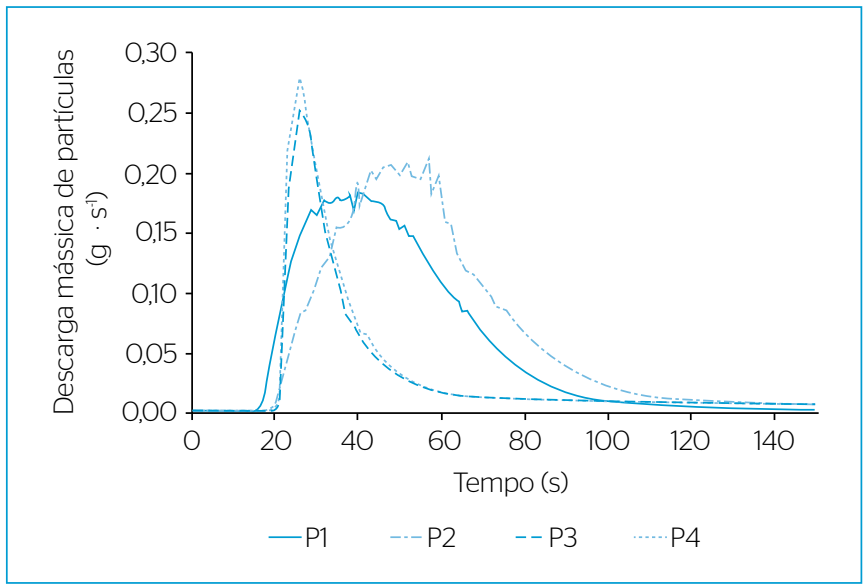

Figura 6 - Polutogramas médios observados para as quatro posições estudadas (P1, P2, P3 e P4). enquanto, para as posições mais distantes, há a presença de um patamar, semelhante ao verificado no transporte de areia. Considerando o instante em que ocorre o pico da descarga como o início do patamar para os casos de $\mathrm{P} 1$ e P2, percebe-se um atraso em relação à $\mathrm{P} 3 \mathrm{e}$ P4. Nota-se, ainda, uma diferença entre os polutogramas P1 e P2 não expectável, pois estão à mesma distância do exutório, apenas localizadas em vertentes diferentes da superfície. Isso pode ser explicado pela diferença da intensidade de precipitação e da distribuição espacial da precipitação nas posições P1 e P2 (Figura 2). Na posição 1, a intensidade média de precipitação é menor se comparada à posição 2 , porém a distribuição espacial da precipitação em P1 (CUC=86\%) é mais uniforme do que na posição P2 (CUC=77\%).

$\mathrm{O}$ valor de pico da descarga mássica obtido para P3 e P4 (0,26 e 0,27 g.s $\left.\mathrm{s}^{-1}\right)$ é maior em relação a P1 e P2 (0,18 e 0,21 g.s . $\left.^{-1}\right)$, mas essa diferença é menor do que aquela observada no transporte de partículas. Observa-se na Tabela 1 que o transporte de material em suspensão para P1 e P2 é mais lento do que o transporte do material dissolvido para estas mesmas posições, contudo as velocidades de transporte de material em suspensão e material dissolvido foram semelhantes para P3 e P4. Isso pode ser explicado pelo fato de que o transporte de poluentes em suspensão é dependente da energia do escoamento, que, neste estudo, é quase nulo próximo às posições $\mathrm{P} 1$ e P2, sendo crescente ao longo da calha coletora devido ao regime variado do escoamento.

$\mathrm{O}$ adiantamento do tempo de pico em P3 e P4 em relação a P1 e $\mathrm{P} 2$, verificado nos particulogramas e polutogramas, deve-se ao fato de: - a distância do centro de massa $(0,7 \mathrm{~m})$ de P3 e P4 ao exutório é menor do que a distância do centro de massa P1 e P2 (3,5 m) ao exutório;

- nas posições $\mathrm{P} 1$ e P2, os poluentes estão sujeitos principalmente ao efeito da precipitação, enquanto os poluentes que estão nas posições P3 e P4 sofrem o efeito da precipitação e da altura do escoamento.

A combinação destes dois fatores faz com que os poluentes que estão mais próximos do exutório sejam mais rapidamente transportados, resultando nos picos mais pronunciados.

Na comparação entre particulogramas e polutogramas, nota-se que as posições mais próximas do exutório (P3 e P4) apresentaram tempo

Tabela 1 - Valores de tempos de pico (s), descarga mássica máxima de areia e de cloreto de sódio $\left(\mathrm{g} \cdot \mathrm{s}^{-1}\right)$ encontrados para hidrogramas, particulogramas e polutogramas para as regiões avaliadas (P1, P2, P3 e P4).

\begin{tabular}{l|c|c|c|c|c}
\multirow{2}{*}{ Posição } & \multicolumn{2}{|c|}{ Tempo de pico } & $\begin{array}{c}\text { Descarga } \\
\text { máxima de areia }\end{array}$ \\
\cline { 2 - 6 } Descarga máxima \\
de cloreto de sódio
\end{tabular}


de pico mais reduzidos para o transporte dos poluentes em suspensão e dissolvidos e, consequentemente, resultaram em maiores valores de pico da descarga mássica (maior concentração das partículas de areia e do cloreto de sódio no escoamento). Para as posições mais distantes do exutório (P1 e P2), os instantes em que ocorreram o valor do pico foram distintos para o cloreto de sódio e para as partículas de areia, porém, quando a posição da fonte de poluente está mais próxima do exutório, os instantes em que ocorrem os picos para o transporte de cloreto de sódio e de areia são aproximadamente iguais. Isso evidencia que o transporte de poluentes em suspensão se aproxima do transporte de poluentes solúveis quando as fontes de poluição estão próximas do exutório, possivelmente devido ao fato de que a energia do escoamento nessas posições é suficiente para arrastar as partículas em suspensão de maneira análoga aos poluentes dissolvidos no escoamento.

$\mathrm{Na}$ Figura 7 são apresentadas as relações entre os tempos de pico dos particulogramas (Tsus) e dos polutogramas (Tdis) com o tempo de pico do hidrograma (Th). Para os particulogramas, os tempos de pico foram definidos no momento em que foi alcançado o valor máximo da massa transportada, enquanto, para os polutogramas, o tempo de pico foi definido no instante em que a concentração de sólidos totais dissolvidos atingiu o valor do patamar; nos hidrogramas, o tempo de pico considerado foi igual a 31,0 segundos para todos os ensaios, pois a partir desse instante, as vazões são caracterizadas pelo patamar de equilíbrio.

Pode-se observar, a partir de uma análise da Figura 7 e dos dados da Tabela 1, que a relação Tsus/Th diminui com as partículas de areia se aproximando do exutório, enquanto a relação Tdis/Th aumenta com as partículas de cloreto de sódio dispostas mais próximas do exutório (P3 e P4). Para as posições $\mathrm{P} 1$ e P2, a diferença entre o transporte de areia e de cloreto de sódio é evidente, pois a relação de Tsus/Th é igual a 4,44 (P1) e 3,40 (P2), enquanto Tdis/Th é igual a 0,29 (P1) e 0,48 (P2). Para estas posições, o transporte de cloreto de sódio é muito mais rápido, quando comparado ao da areia. Para as posições P3 e P4, a diferença diminui significativamente, sendo Tsus/Th igual a 0,84 (P3) e 0,77 (P4) e Tdis/Th igual a 1,02 (P3) e 1,09 (P4). Nova evidência de que se as fontes de poluição estão mais próximas do exutório, o transporte de cloreto de sódio e de areia possuem características semelhantes, devido ao fato de que nas posições 3 e 4 há uma lâmina de escoamento maior do que a existente em P1 e P2.

Na Figura 8 é apresentada a relação entre a massa acumulada normalizada de poluente transportado e o volume acumulado normalizado de escoamento superficial. A massa acumulada normalizada $\left(M^{*}\right)$ é a relação entre a massa transportada acumulada até um determinado

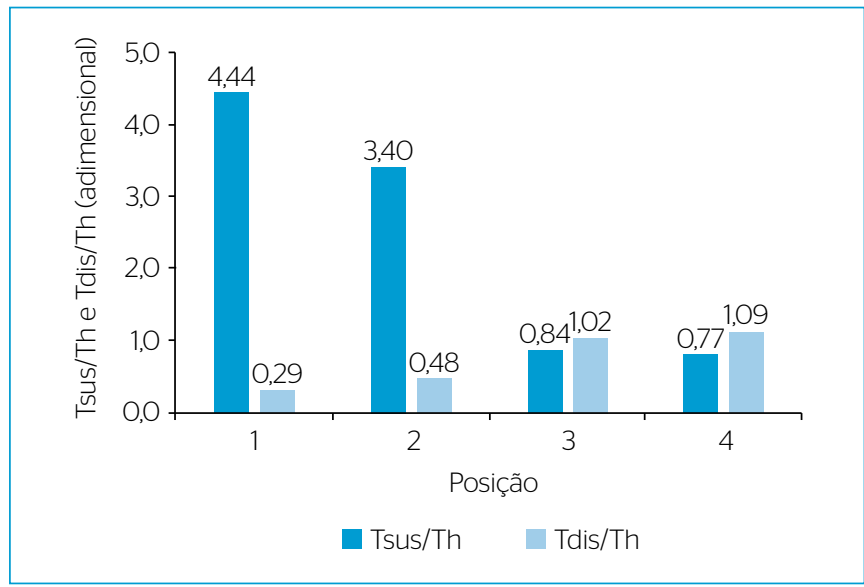

Figura 7 - Relação entre o tempo de pico para os particulogramas (Tsus) e polutogramas (Tdis) em relação ao tempo de pico dos hidrogramas (Th) para as quatro posições (P1, P2, P3 e P4).

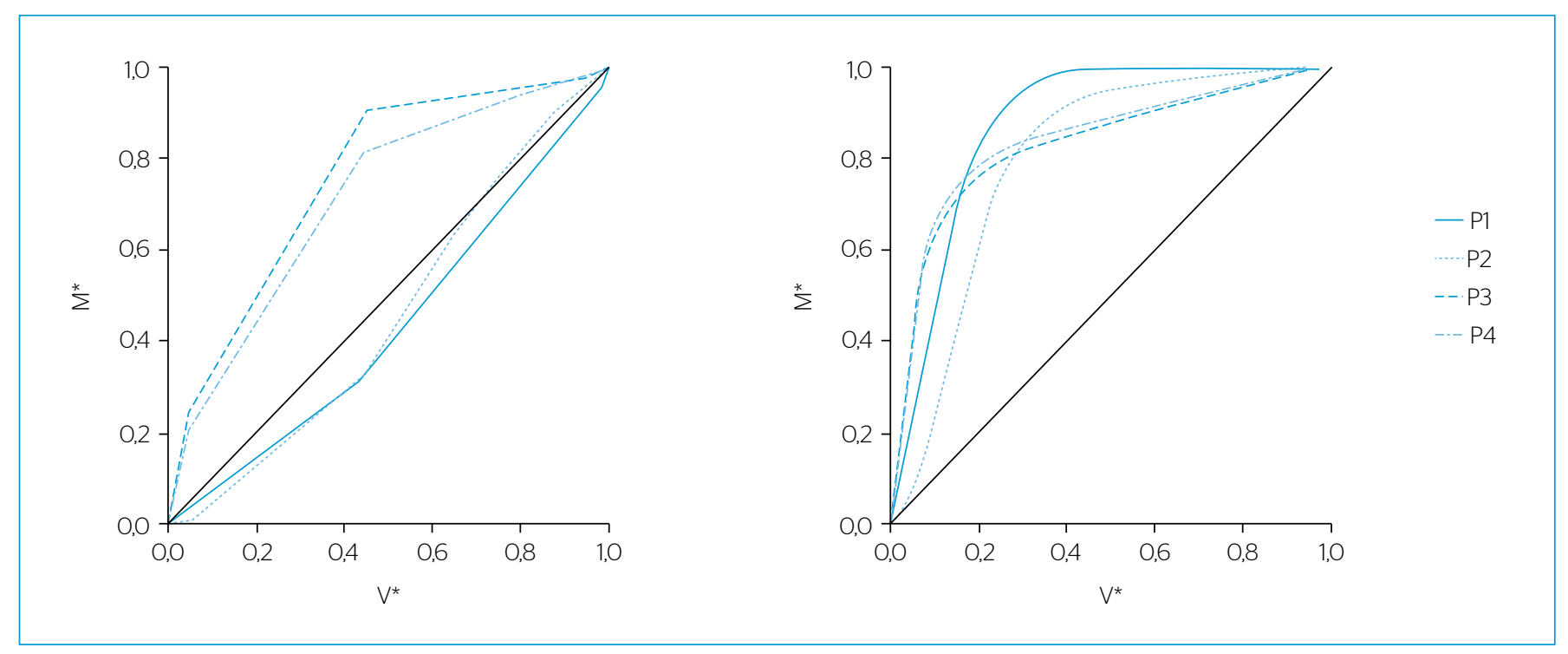

Figura 8 - Relação entre a massa acumulada normalizada do material em suspensão (direita) e dissolvido (esquerda) e o volume acumulado normalizado. 
instante e a massa total transportada. O volume acumulado normalizado $\left(\mathrm{V}^{*}\right)$ é a relação entre o volume escoado acumulado até um determinado instante e o volume total escoado. O conceito de Mass First Flush (MFF) foi utilizado para quantificar a massa de partículas transportadas e pode ser calculada em qualquer ponto da curva, dividindo-se a massa acumulada normalizada das partículas transportadas pelo escoamento acumulado normalizado $\left(M F F=\frac{M^{\star}}{V^{\star}}\right)$. Por definição, o MFF é igual a zero no início do evento de precipitação e sempre igual a 1,0 no final. Valores superiores a 1,0 indicam que as partículas são lavadas mais rapidamente do que o escoamento, indicando a existência de um efeito de first flush.

Observa-se na Figura 8 que o transporte de material em suspensão é distinto para as posições mais próximas e mais distantes do exutório. Para $\mathrm{P} 1$ e $\mathrm{P} 2$, o transporte de partículas é igual a $\mathrm{MFF}<1$, enquanto para P3 e P4, MFF>1, evidenciado o efeito de first flush nestas últimas posições. O transporte de material dissolvido apresentou comportamento mais uniforme independentemente da posição da fonte de poluição, pois, em todas as posições, MFF $>1$ evidencia forte efeito de first flush. Para o transporte de material dissolvido observa-se que, por exemplo, para $20 \%$ do escoamento superficial ocorrido, 70 a $80 \%$ da massa de cloreto de sódio já passou pelo exutório (MFF 4).

\section{CONCLUSÕES}

Foram realizados experimentos em laboratório para investigar a influência da localização da fonte difusa em relação ao exutório da área de drenagem sobre o transporte de poluentes, nomeadamente representados neste artigo pela areia fina e pelo cloreto de sódio, simulando material particulado e dissolvido, respectivamente, sob condições de chuva artificial em superfície impermeável. A análise dos resultados dos experimentos, realizada a partir dos valores médios de cinco experimentos para cada posição, permitiu concluir que há diferenças entre o transporte desses materiais pelo escoamento superficial. As seguintes conclusões podem ser apresentadas:

- as partículas de areia fina dispostas na superfície acrílica não interferem na forma dos hidrogramas independentemente de sua localização na superfície;

- o instante em que ocorre o pico do transporte de poluentes dissolvidos ou em suspensão é o mesmo quando a fonte de poluição está localizada próxima ao exutório, evidenciando uma semelhança no comportamento para ambos materiais;

- o transporte de poluente dissolvido é muito mais rápido do que o transporte de partículas em suspensão quando a fonte está mais afastada do exutório;

- no transporte de poluentes dissolvidos verifica-se o efeito de first flush durante todo o escoamento independentemente da posição em que foi distribuído o cloreto de sódio. Já no transporte de partículas em suspensão, o efeito de first flush é verificado somente nas posições mais próximas ao exutório.

Os resultados experimentais indicaram diferenças no transporte de poluentes dissolvidos e em suspensão, evidenciando a importância desta temática para estudos sobre a gestão das águas urbanas, a fim de encontrar soluções acerca do tratamento adequado para os impactos que podem decorrer da localização de atividades com potencial poluidor em relação à rede de drenagem. Estudos mais detalhados podem ser feitos com outros tipos de poluentes, novas localizações das fontes poluidoras, outras declividades e materiais da superfície.

\section{AGRADECIMENTOS}

Este trabalho foi realizado com apoio financeiro da Coordenação de Aperfeiçoamento de Pessoal de Nível Superior (CAPES), projeto 88881.0300412/2013-01.

\section{REFERÊNCIAS}

ABUDI, I.; CARMI, G.; BERLINER, P. (2012) Rainfall simulator for field runoff studies. Journal of Hydrology, v. 454-455, p. 76-81. DOI: 10.1016/j.jhydrol.2012.05.056

AKSOY, H.; UNAL, N.E.; COKGOR, S.; GEDIKLI, A.; YOON, J.; KOCA, K.; BORAN, S.; ERIS, E. (2012) A rainfall simulator for laboratoryscale assessment of rainfall-runoff-sediment transport processes over a two-dimensional flume. Catena, v. 98, p. 63-72. DOI: 10.1016/j. catena.2012.06.009
ALI, S.A.; BONHOMME, C.; CHEBBO, G. (2016) Evaluation of the performance and the predictive capacity of build-up and wash-off models on different temporal scales. Water, v. 8, n. 312. http://dx.doi. org/10.3390/w8080312

BI, E.G.; MONETTE, F.; GASPERI, J. (2015) Analysis of the influence of rainfall variables on urban effluents concentrations and fluxes in wet weather. Journal of Hydrology, v. 523, p. 320-332. DOI: 10.1016/j. jhydrol.2015.01.017 
CARVALHO, S.C.P. (2014) Rainfall: measurements, variability and laboratory simulations. 216f. Tese (Doutorado em Engenharia) Universidade de Coimbra, Coimbra.

CASTRO, A.S.; GOLDENFUM, J.A.; SILVEIRA, A.L. da; MARQUES, D. da M. (2013) Avaliação da evolução do comportamento quantitativo de pavimentos permeáveis no controle do escoamento superficial. Revista Brasileira de Recursos Hídricos, v. 18, n. 1. DOI: 10.21168/rbrh. v18n1.p263-273

CHRISTIANSEN, J.E. (1941) The uniformity of application of water by sprinkler systems. Agricultural Engineering, v. 22, p. 89-92.

CLARKE, M.A.; WALSH, R.P.D. (2007) A portable rainfall simulator for field assessment of splash and slopewash in remote locations. Earth Surface Processes and Landforms, v. 32, n. 13, p. 2052-2069. DOI: 10.1002/esp.1526

DENG, Z.; LIMA, J.L.M.P.; SINGH, V.P. (2005) Transport rate-based model for overland flow and solute transport: parameter estimation and process simulation. Journal of Hydrology, v. 315, p. 220-235. DOI: 10.1016/j.jhydrol.2005.03.042

EGODAWATTA, P.; THOMAS, E.; GOONETILLEKE, A. (2009) Understanding the physical processes of pollutant build-up and wash-off on roof surfaces. Science of the Total Environmental, v. 407, n. 6. p. 1834-1841. https://doi.org/10.1016/j.scitotenv.2008.12.027

FLETCHER, T.D; ANDRIEU, H.; HAMEL, P. (2013) Understanding, managementand modelling of urban hydrology and its consequences for receiving waters: a state of the art. Advances in Water Resources, v. 51, p. 261-279. https://doi.org/10.1016/j.advwatres.2012.09.001

GOMES, K.M.F. (2014) Modelagem da poluição difusa em uma bacia de drenagem urbana. 76 f. Dissertação (Mestrado em Engenharia Sanitária) - Universidade Federal do Rio Grande do Norte, Natal.

GRIMM, N.B.; FAETH, S.H.; GOLUBIEWSKI, N.E.; REDMAN, C.L.; WU, J.; BAI, X.; BRIGGS, J.M. (2008) Global change and the ecology of cities. Science, v. 319, p. 756-760. DOI: 10.1126/science.1150195

HERNÁNDEZ, R. del P.B. (2009) Estudo do comportamento de cobre em águas de chuva sintéticas de São Paulo e Rio de Janeiro e sua proteção contra corrosão por revestimentos e pátinas expostos à ação climática. Tese (Doutorado em Engenharia) Universidade de São Paulo, São Paulo.

ISERLOH, T.; RIES, J.B.; ARNÁEZ, J.; BOIX-FAYOS, C.; BUTZEN, V.; CERDÀ, A.; ECHEVERRÍA, M.T.; FERNÁNDEZ-GÁLVEZ, J.; FISTER, W.; GEIßLER, C.; GÓMEZ, J.A.; GÓMEZ-MACPHERSON, H.; KUHN, N.J.; LÁZARO, R.; LEÓN, F.J.; MARTÍNEZ-MENA, M.; MARTÍNEZMURILLO, J.F.; MARZEN, M.; MINGORANCE, M.D.; ORTIGOSA, L.; PETERS, P.; REGÜÉS, D.; RUIZ-SINOGA, J.D.; SCHOLTEN, T.; SEEGER, M.; SOLÉ-BENET, A.; WENGEL, R.; WIRTZ, S. (2013) European small portable rainfall simulators: A comparison of rainfall characteristics. Catena, v. 110, p. 100-112. https://doi.org/10.1016/j. catena.2013.05.013

KEMERICH, P.D. da C.; MARTINS, S.R.; KOBIYAMA, M.; SANTI, A.L.; FLORES, C.E.B.; BORBA, W.F. de; FERNANDES, G.D.; CHERUBIN, M.R. (2O13) Qualidade da água oriunda do escoamento superficial simulado em bacia hidrográfica. Revista Ciência e Natura, v. 35, n. 2. htp://dx.doi.org/10.5902/2179-460X849
KIM, L.; KAYHANIAN, M.; ZOH, K.; STENSTROM, M.K. (2OO5) Modeling of highway stormwater runoff. Science of the Total Environmental, v. 348, p. 1-18. DOI: 10.1016/j.scitotenv.2004.12.063

LEDFORD, S.H.; LAUTZ, L.K. (2015) Floodplain connection buffers seasonal changes in urban stream water quality. Hydrological Processes, v. 29, n. 6, p. 1002-1016. DOI: 10.1002/hyp.10210

LIMA, E.B.N.R. (2001) Modelação integrada para a gestão da qualidade da água na bacia do Rio Cuiabá. 184 f. Tese (Doutorado em Ciências em Engenharia Civil) - Universidade Federal do Rio de Janeiro, Rio de Janeiro.

LIMA, J.L.M.P.; SOUZA, C.C.S.; SINGH, V.P. (2008) Granulometric characterization of sediments transported by surface runoff generated by moving storms. Nonlinear Processes in Geophysics, v. 15, p. 999-1011.

LIU, Y:; AHIABLAME, L.M.; BRALTS, V.F.; ENGEL, B.A. (2015) Enhancing a rainfall-runoff model to assess the impacts of BMPs and LID practices on storm runoff. Journal of Environmental Management, v. 147, p. 12-23. https://doi.org/10.1016/j.jenvman.2014.09.005

MILLER, J.D.; KIM, H.; KJELDSEN, T.R.; PACKMAN, J.; GREBBY, S.; DEARDEN, R. (2014) Assessing the impact of urbanization on storm runoff in a peri-urban catchment using historical change in impervious cover. Journal of Hydrology, v. 515, p. 59-70. https://doi. org/10.1016/j.jhydrol.2014.04.011

MORAES, I.C.; CONCEIÇÃO, F.T. da; CUNHA, G.M.L. da; MORUZZI, R.B. (2014) Comparação de metodologias na definição de fluxos acumulados a partir de modelos digitais de elevação de terreno aplicado a suscetibilidade de inundações. Revista Brasileira de Recursos Hídricos, v. 19, n. 2.

MUTCHLER, C.K.; MOLDENHAUER, W.C. (1963) Applicator for laboratory rainfall simulator. Transactions of the ASAE, v. 6, n. 3, p. 220-222. DOI: 10.13031/2013.40871

NASCIMENTO, N.O; HELLER, L. (2005) Ciência, tecnologia e inovação na interface entre as áreas de recursos hídricos e saneamento. Engenharia Sanitária e Ambiental, v. 10, n. 1, p. 36-48. http://dx.doi.org/10.1590/S1413-41522005000100005

O'DRISCOLL, M.; CLINTON, S.; JEFFERSON, A.; MANDA, A.; MCMILLAN, S. (2010) Urbanization effects on Watershed Hydrology and In-stream Processes in the Southern United States. Water, v. 2, n. 3, p. 605-648. DOl: 10.3390/w2030605

OMETO, J.P.H.B.; MARTINELLI, L.A.; BALLESTER, M.V.; GESSNER, A.; KRUSCHE, A.V.; VICTORIA, R.L.; WILLIANS, M. (2000) Effects of land use on water chemistry and macroinvertebrates in two streams of the Piracicaba river basin, south-east Brazil. Fresh Water Biology, n. 44, p. 327-337. DOI: 10.1046/j.1365-2427.2000.00557.x

PAUL, M.J.; MEYER, J.L. (2001) Streams in the urban landscape. Annual Review of Ecology and Systematics, v. 32, p. 333-365. https:// doi.org/10.1146/annurev.ecolsys.32.081501.114040

PINHEIRO, A.; KAUFMANN, V.; SCHNEIDERS, D; GOTARDO, R (2013) Transporte de sedimentos e espécies químicas em áreas de reflorestamentos e pastagem com base em chuva simulada. Revista Ambiente \& Água, v. 8, n. 2. http://dx.doi.org/10.4136/ambi-agua.1106 
POLETO, C. (Org.). (2008) Ambiente e sedimentos. Porto Alegre: ABRH. 404 p.

REIS, S.S. dos. (2015) Influência da densidade e da altura de edificações na transformação de precipitação-escoamento utilizando chuva simulada em modelo reduzido de bacia hidrográfica urbana com diferentes declividades. 94 f. Dissertação (Mestrado em Ciência e Engenharia Ambiental) - Universidade Federal de Alfenas, Poços de Caldas.

SILVEIRA, A.; ISIDORO, J.M.P.G.; DEUS, F.P.; REIS, S.S.; SILVA, A.M.; GONÇALVES, F.A.; MENEZES, P.H.B.J.; TIEZZI, R. (2017) Enhancing the spatial rainfall uniformity of pressurized nozzle simulators. Management of Environmental Quality, v. 28, n. 1. https://doi. org/10.1108/MEQ-07-2015-0140

SPOHR, R.B.; CORCINI, A.L.M.; PELLEGRIN, J.; BONFANTI, J.B.; SOTO, M.F.D.; CARDOSO, T. (2015) Desenvolvimento e validação de um simulador de chuvas portátil. Revista Brasileira de Recursos Hidricos, v. 20, n. 2 .
TANG, J.Y.M.; ARYAL, R.; DELETIC, A.; GERNJAK, W.; GLENN, E.; MCCARTHY, D.; ESCHER, B. (2013) Toxicity characterization of urban stormwater with bioanalytical tools. Water Research, v. 47, p. 5594-5606. DOI: 10.1016/j.watres.2013.06.037

UNITED NATIONS DEPARTMENT OF ECONOMIC AND SOCIAL AFFAIRS. (2011) Population distribution, urbanization, internal migration and development: an international perspective. Nova York: United Nations Department of Economic and Social Affairs. 378 p

VAZE, J.; CHIEW, F.H.S. (2003) Study of pollutant wash off from small impervious experimental plots. Water Resources Research, v. 39, n. 6. DOI: 10.1029/2002WR001786

VINAGRE, M.V.A.; LIMA, A.C.M.; LIMA JUNIOR, D.L. (2015) Estudo do comportamento hidráulico da Bacia do Paracuri em Belém (PA) utilizando o programa Storm Water Management Model. Engenharia Sanitária e Ambiental, v. 20, n. 3. http://dx.doi. org/10.1590/S1413-41522015020000106515 